\title{
Contribution à l'étude du régime alimentaire, de la croissance et de la fécondité de la perche (Perca fluviatilis L.) dans un lac oligotrophe de région tempérée Contribution to the study of the food, of the growth and of the fecondity of the perch (Perca fluviatilis L.) in a temperate oligotropic lake
}

\author{
R. Chappaz, G. Brun et G. Olivar
}

Volume 2, numéro 1, 1989

URI : https://id.erudit.org/iderudit/705025ar

DOI : https://doi.org/10.7202/705025ar

Aller au sommaire du numéro

Éditeur(s)

Université du Québec - INRS-Eau, Terre et Environnement (INRS-ETE)

ISSN

0992-7158 (imprimé)

1718-8598 (numérique)

Découvrir la revue

Citer cet article

Chappaz, R., Brun, G. \& Olivar, G. (1989). Contribution à l'étude du régime alimentaire, de la croissance et de la fécondité de la perche (Perca fluviatilis L.) dans un lac oligotrophe de région tempérée. Revue des sciences de l'eau/ Journal of Water Science, 2(1), 95-107. https://doi.org/10.7202/705025ar

\section{Résumé de l'article}

La retenue de Sainte-Croix sur te Verdon, mise en eau il y a dix ans, est un lac oligotrophe monomictique chaud de 2200 ha de surface et de $80 \mathrm{~m}$ de profondeur maximum (profondeur moyenne $50 \mathrm{~m}$ ). Une vidange hivernale partielle en abaisse le niveau de 8 à $16 \mathrm{~m}$ selon les années. La température dans l'épilimnion varie de $7{ }^{\circ} \mathrm{C}$ en février à $22^{\circ} \mathrm{C}$ en juillet, avec une moyenne de plus de 900 degrés/jours au-dessus de $14^{\circ} \mathrm{C}$.

Les perches introduites depuis la mise en eau, se nourrissent des plus gros planctontes (Cladocères et Copépodes), des larves benthiques de divers insectes (Chironomides, Ephémères) ainsi que d'alevins. Malgré des conditions thermiques favorables, la croissance des perches est lente en raison de la faible densité des proies et de la concurrence avec des Cyprinidae planctonophages, ablettes et gardons, très bien représentés dans la retenue.

La maturité sexuelle est atteinte au terme de ta première année pour la majorité des mâles, la seconde année seulement pour $60 \%$ des femelles. Mesurée sur 15 femelles de 14 à $40 \mathrm{~cm}$, la fécondité variant de 2500 à 50000 ovules, est faible. 


\section{Contribution à l'étude du régime alimentaire, de la croissance et de la fécondité de la perche (Perca fluviatilis $L$.) dans un lac oligotrophe de région tempérée}

Contribution to the study of the food, of the growth and of the fecondity of the perch (Perca fluviatilis L.) in a temperate oligotropic lake

R. CHAPPAZ, G. BRUN, G. OLIVARI (1)

RÉSUMÉ

La retenue de Sainte-croix sur le Verdon, mise en eau il y a dix ans, est un lac oligotrophe monomictique chaud de 2200 ha de surface et de $80 \mathrm{~m}$ de profondeur maximum (profondeur moyenne $50 \mathrm{~m}$ ). Une vidange hivernale partielle en abaisse le niveau de 8 à $16 \mathrm{~m}$ selon les années. La température dans l'épilimnion varie de $7{ }^{\circ} \mathrm{C}$ en février a $22^{\circ} \mathrm{C}$ en juillet, avec une moyenne de plus de 900 degrés/jours au-dessus de $14{ }^{\circ} \mathrm{C}$.

Les perches introduites depuis la mise en eau, se nourrissent des plus gros planctontes ( $\mathrm{Cladocères} \mathrm{et} \mathrm{Copépodes),} \mathrm{des}$ larves benthiques de divers insectes (Chironomides, Ephémères) ainsi que d'alevins. Malgré des conditions thermiques favorables, la croissance des perches est lente en raison de la faible densité des proies et de la concurrence avec des Cyprinidae planctonophages, ablettes et gardons, très bien représentés dans la retenue.

La maturité sexuelle est atteinte au terme de la première année pour la majorité des mâles, la seconde année seulement pour $60 \%$ des femelles. Hesurée sur 15 femelles de 14 à $40 \mathrm{~cm}$, la fécondité variant de 2500 à 50000 ovules, est faible.

Mots clès : retenue artificielle, perche, croissance, alimentation, féconditê, lac de Ste-Croix. 
The Large-scale hydro-electric reservoir of Lake Sainte-croix fed by the verdon river, was dammed in 1974 and holds $765 \mathrm{hm}^{3}$, spreading over 2200 ha at 477 meters above sea level. Its average and maximum depths are 50 and 80 meters and the water Level can fall 16 meters in Autumn and Winter, reducing the water cover by a maximum of 23 \% during January and February. It is a warm monomictic lake where the hypolimnion is well oxygenated in all seasons. The water has an alkaline $\mathrm{pH}$ and is retatively poor in nutritional salts. Controlled by water temperature during the mixing period, the primary production, always low, is limited by loss of nitrogen in the epilimnion and lack of light in the hypolimnion during the thermal stratified period.

The epil-imnion temperature varies from $7{ }^{\circ} \mathrm{C}$ in february to $22{ }^{\circ} \mathrm{C}$ in July, with an average of more than 900 degrees per day per year above $14^{\circ} \mathrm{C}$. In addition to the various indigenous species, perch, roach and bleak are stocked, the latter two species being predominant now. The stomach contents of perch fished with nets and by electrical means during the winter (December-March) and Summer (August) periods were examined and the animals' ages were determined using the opecularbone method. The scarcity of plankton compels the young as well as the adult perch to feed on macroinvertebrates of benthos. This trophic niche overlap between the two size groups creates intraspecific competition. The availability of benthic prey is conditioned to a great extent by the receeded water line in Winter (winter tide) which leaves the essential feeding ground dry for several months and $\mathrm{l} i m i$ ts the development of aquatic vegetation. The existence of an interspecific roach-bleak competition, especially noticeable between the young planktivorous fry, accentuates the effects of the poor nutritional conditions on the rate of perch reproduction which is among the lowest on record. Sexual maturity is reached at the end of the first year for the males, and during the second year for only $50 \%$ of the females. The absolute fertility mesured on 15 females between 14 and $40 \mathrm{~cm}$, varied from 2,500 to 50,000 eggs.

Key-words : perch, growth rate, feeding habits, fecondity, hydroelectric reservoir.

\section{INTRODUCTION}

La perche, très bien développée dans les lacs et rivières d'Europe, étend son aire de répartition vers le sud en raison de son introduction dans les retenues artificlelies.

Pourtant, le devenir des perches introduites dans les lacs de barrage de grande dimension est mal connu. HOLCIK, (1966), indique qu'une population de perches se développe rapidement à la mise en eau du réservoir Klicava, puis voit ses effectifs diminuer quelques annés plus tard. 
Plusieurs facteurs semblent contrôlex la dynamique des populations de perches : la température et la densité de la population (LE CREN, 1947 ; HOESTLAND, 1979 ; BOUJARD, 1987), l'abondance du zooplancton et des invertébrés (LE CREN, 1947 ; CRAIG, 1974 ; THORPE, 1977 ; PERSSON, 1983), le statut trophique de la retenue (HARIMAN, 1977) et la concuxrence avec d'autres espèces et notamment le gardon (LESSMARK, 1983).

Mise en eau en 1974, le lac de Sainte-Croix est une retenue hydroélectrique de grande capacité : $765 \mathrm{hm}^{3}$ pour une surface de 2200 ha à la côte normale de remplissage, $477 \mathrm{~m}$, qui se malntient 5 a 6 mois, de juin à novembre. La profordeur maximum est de $80 \mathrm{~m}$, la profondeur moyenne de $50 \mathrm{~m}$. Le niveau du plan d'eau peut s'abaisser jusqu'à la cote $461 \mathrm{~m}$, soit $16 \mathrm{~m}$, au cours de l'automne et de l'hiver = la surface inondée subit ainsi une réduction maximum de 23 \& en janviex-février. Sainte-croix est un lac monomictique chaud dont l'hypolimnion est bien oxygéné en toutes saisons. Les eaux ont un $\mathrm{pH}$ basiques, elles sont relativement pauvres en sels nutritifs (CBAypeau, 1982). Pendant la période de brassage la température limite la production primaire à des valeurs très basses : 3 à $8 \mathrm{mg} / \mathrm{c} / \mathrm{m}^{2} / \mathrm{h}$. Le maximum est atteint au début de la période de réchauffement de $l^{\prime}$ eau avec $46,9 \mathrm{mg} / \mathrm{c} / \mathrm{m}^{2} / \mathrm{h}$ au mois de mai. En période stratifíe la production primaire dans l'épilimnion est faible à la suite de l'épuisement progressif des sels nutritifs, alors que dans I'hypolimnion peu ou pas productif, faute de lumière, la teneur en nitrates augmente au cours de l'été par minéralisation de la matière organique qui sedimente. Les orthophosphates restent toute $l$ 'année a un niveau très bas, toujours inférieur à $1 \mathrm{\mu g} / 1$ (EL HAFA, 1987). La retenue de Ste-Crolx présente, toutes les caractéristiques d'un lac oligotrophe, équilibré, de région tempérée.

Le limnozooplancton est essentiellement composé de Cladocères des genres Daphnia et Ceriodaphnia et du Rotifère Asplanchna priodonta (BRESSAC, 1985).

Dans les bajes peu profondes où s'est implanté un herbier à Myrlophylles et Potamots, se developpe un héléplancton plus diversifié, dominé par les cladocères Simocephalus vetulus, Sida cristallina et le copepode Macrocyclops albidus. Les fonds vaseux sont peuplés d'oligochètes et de chlronomes; les premiers occupent la zone profonde, les seconds la zone littorale. La densité moyenne des ollgochètes est de $6000 \mathrm{ind} / \mathrm{m}^{2}$, celle des Chironomes de $2800 \mathrm{ind} / \mathrm{m}^{2}$. Le benthos de 1 'herbier comporte des larves d'Ephêméroptères, de Trichoptères et d'odonates.

Outre la perche, de nombreuses espéces ont fait l'objet d'introductions ; le gardon et l'ablette ont notamment connus une explosion démographique et sont maintenant dominants, représentant 35 et 20 o de 1 'effectif de nos captures scientifiques. La perche ne représente que 4 \& (CAAPPAZ et al., 1987).

Cet article étudie le régime alimentaire de la perche (Percha fluviatilis $L$. ) de la retenue de Ste-Croix, sa croissance et sa fécondité en relation avec la température, l'oligotrophe de l'écosystème, et la présence d'autres espèces, notamment le gardon et l'ablette. 


\section{2 - Matériel.s et méthode}

Les enregistrements de températures sont effectués par Electricité de France dans l'épilimnion ( 8 à $10 \mathrm{~m}$ de profondeur), les mesures sont effectuées toutes les 3 heuxes. Ces mesures permettent de calculer des moyennes mensuelles, présentées dans la figure 1 , et des moyennes quotidiennes qui permettent de calculer le nombre de

Temp. ${ }^{\circ} \mathrm{C}$

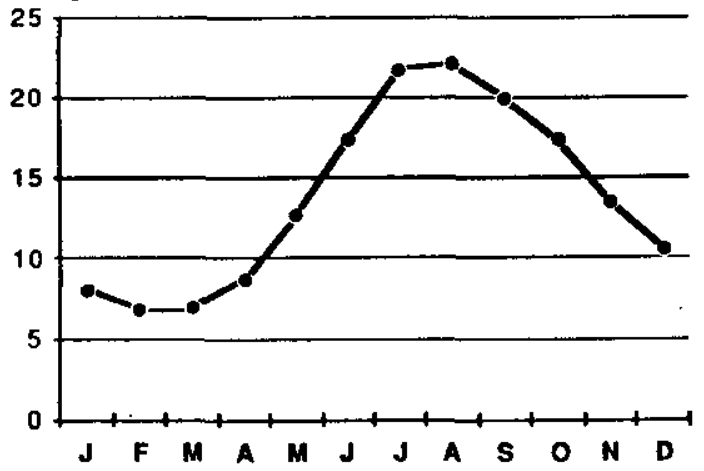

Figure 1.- Temperature dans 1'épilimnion du lac de Sainte-Croix : moyennes mensuelles calculées à partir de relevếs horaires de juin 1982 à juin 1987.

Figure 1.- Temperature in the epilimion of the sainte croix resemoir : monthly means calculated from hourly values from june 1982 to june 1987. degrés-jours au dessus d'une température choisie. Le passage de 10 à $14^{\circ} \mathrm{C}$ demande de 13 à 40 jours selon les années, le nombre moyen de degrés-jours par an au dessus de $14^{\circ} \mathrm{C}$ a été supérieur à 900 pendant notxe période d'étude.

\section{Les filets maillants} sont utilisês pour les captures en zone pxofonde, où ils représentent notre seul moyen d'investigation. Différentes mailles $(14,18$, $22,25,27,32,40$ et $50 \mathrm{~mm}$ ) sont utiliskes pour capturer toutes les classes de taille de la population. Posés dés la tombée de la nuit, ils sont relevés à 1 aube. Cette technique présente cependant quelques inconvénients liés aux risques de régurgitation ou à

la digestion des proies entre le maillage du poisson et sa digestion qui peuvent être séparés de plusieurs heures. Les zones de bordures ont fait 1 'objet te peches électriques, a pieds ou a partir d'un bateau, qui nous ont permis la capture de juvéniles.

Les contenus stomacaux sont fixés au formol à 10 q dès la capture. En rafson de la petite taille des échantillons ils ont été séparés en 2 groupes : celui d'hiver formé des poissons capturés de décembre 1984 à mars 1985 et celui d'été formé des poissons capturés en aout 1984 . L'analyse des contenus stomacaux a donné lieu au calcul de la fréquence relative ( $P($ ) de chaque prole, du pourcentage de la biomasse des proies (Mi), qu'elle représente et de son occurence (Oi).

La fréquence relative (Pi) d'une catégorie de proie (i) dans une série d'estomacs $(1,2, \ldots . j, \ldots$ p) est le rapport entre le nombre de proies de cette catégorie et le nombre total de proies consommées :

$$
P i=\frac{\sum_{j=1}^{p} \times i j}{\sum_{i=1} \sum_{j=1} \times i j} \times 100
$$

où $\mathrm{xij}=$ nombre de projes de la catégorie $i$ dans I'estomac $j$. 
Cette méthode surestime l'importance des petites proies et sous-estime celle des proies digérées le plus rapidement.

On calcule de même Mi en remplaçant les nombres de proies par les biomasses correspondantes.

L'occurrence d'un aliment est exprimée par le pourcentage des estomacs qui contiennent cet aliment :

$$
O i=\frac{E i}{E} \times 100
$$

$E i$ = nombre $d$ 'estomacs contenant $i$

$E$ = nombre d'estomacs contenant une proie quelconque (non vides).

Comme le souligne HYSLOP (1980), l'importance d'une proie dans le régime alimentaire d'une espèce varie suivant l'indice considéré (nombre, biomasse ou occurrence). Nous avons dorc utilisé pour analyser le régime de la perche l'"index of relative importance" (IRI) (PINKAS et $a$ l., 1971 in HYSLOP, 1980), qui combine les valeurs des fréquences en nombre, en biomasse et I'occurrence :

$$
I R I=(P i+M i) \times O i
$$

Les individus sont mesurés à la fourche au mm près et pesés au gramme près. Le rapport de la taille à la fourche sur la taille totale est de $0,92$ ( $n=126, r=0,96)$.

Ia croissance des individus a été calculée après la détermination de 1'agge. L'age de chacun des individus capturés en hiver (126 au total) a été déterminé par la méthode operculalre, préconisée par LE CREN (1947). Ia lecture se fait sous la loupe binoculaire munie d'un objectif micrométxique. La méthode de LEA, modifiée par LE CREN (1947) a été utilisée pour le rétrocalcul de la taille

$$
\log L f_{t}=\log L f+b\left(\log O_{t}-\log 0\right)
$$

Lf et 0 sont les tailles actuelles du poisson et de son opercule Lft et Ot sont les tailles du poisson et de son opercule au temps $t$.

Pour tous les poissons capturés, une relation entre la taille (Lf) et le poids ( $P$ ) a été calculée à partir du modèle :

$\log P=\log a+b \log L f$

où à et b sont deux paramètres.

Les ovaires ont été prélevés entièrement chez les petits individus et par fragments de 15 à $20 \mathrm{~g}$ chez les plus grands; la conservation se fait dans le liquide de GILSON modiflé par SIMPSON (1951), qui permet également la dissociation des ovocytes. On a calculé la relation liant la fécondité absolue (nombre d'ovocytes) å la longueur des femelles (GOLDSPINK, 1979). 


\section{3 - RÉsultats}

\section{1 - Alimentation}

Nous avons distingué deux catégories d'individus (tableau 1) : ceux de longueur comprise entre 4 et $10 \mathrm{~cm}$, Lf $\leqslant 10 \mathrm{~cm}$, et ceux de longueur comprise entre 13 et $45 \mathrm{~cm}$, Lf $>10 \mathrm{~cm}$.

Tableau 1.- Composition des contenus stomacaux des perches capturées dans le lac de Sainte-Croix. Pi, Mi, Oi, sont respectivement les pourcentages en nombre, en biomasse et en occurrence, des différents types de proies.

Table 1.- Stomach contents of perch in the Sainte-Croix reservoir. $P_{i}, M i$, $D_{i}$ are respectively number frequency, weight frequency and occurence frequency of different prey categories.

\begin{tabular}{|c|c|c|c|c|c|c|c|c|c|c|c|c|}
\hline & \multicolumn{3}{|c|}{$\begin{array}{cc} & 1 \\
\mathrm{Lf} & \leqslant 10 \mathrm{~cm} \\
\mathrm{n}=75\end{array}$} & \multicolumn{3}{|c|}{$\begin{array}{c}\mathbf{E} \quad \mathbf{R} \\
\mathrm{Lf}>10 \mathrm{~cm} \\
\mathrm{n}=45\end{array}$} & \multicolumn{3}{|c|}{$\begin{array}{c}\mathrm{Lf} \leqslant 10 \mathrm{~cm} \\
\mathrm{n}=17\end{array}$} & \multicolumn{3}{|c|}{$\begin{array}{c}\mathbf{E}>10 \mathrm{~cm} \\
\mathrm{n}=22\end{array}$} \\
\hline & $\mathrm{Pi}$ & $\mathbf{M i}$ & oi & $\mathrm{Pi}$ & $\mathrm{Mi}$ & a & $\mathrm{Pi}$ & Mi & $\mathrm{O}$ & $\mathrm{Pj}$ & Mi & $\mathrm{O}$ \\
\hline $\begin{array}{l}\text { Oligochetes } \\
\text { Crustacés }\end{array}$ & 0,54 & - & 1,30 & 0,27 & - & 0,30 & 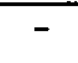 & - & - & - & - & - \\
\hline Cladocères & 25,45 & 1,00 & 51,00 & 0,40 & _ & 8,80 & 7,70 & 0,15 & 12,00 & - & - & - \\
\hline $\begin{array}{l}\text { Copèpodes } \\
\text { Ostracodes }\end{array}$ & $\begin{array}{c}29,75 \\
0,80\end{array}$ & 0,20 & $\begin{array}{c}40,00 \\
660\end{array}$ & $\begin{array}{l}1,70 \\
0,10\end{array}$ & - & $\begin{array}{l}8,80 \\
3,00\end{array}$ & 40 & & 46,00 & - & - & - \\
\hline Amphipodes & $\begin{array}{l}0,30 \\
0,30\end{array}$ & $2, \overline{0} 0$ & $\begin{array}{l}6,60 \\
5,40\end{array}$ & $\begin{array}{l}0,10 \\
2,30\end{array}$ & 1,12 & 17,00 & $0, \overline{0} 2$ & $0, \overline{09}$ & $6, \overline{0} 0$ & $0, \overline{8} 0$ & $0, \overline{50}$ & $\overline{5}$ \\
\hline Isopod & 0,10 & 0,40 & 2,70 & 0,40 & 1,30 & 3,00 & - & - & - & 0,16 & 0,06 & 5 \\
\hline $\begin{array}{l}\text { Decapodes } \\
\text { Insectes }\end{array}$ & - & - & - & 0,30 & 5,60 & 5,60 & - & - & - & - & - & - \\
\hline Odonates & 0,30 & 1,20 & 6,60 & 0,20 & 0,05 & 5,60 & 0,80 & 2,00 & 6,00 & 0,20 & 0,07 & 15 \\
\hline & 1,10 & 2,20 & 6,60 & 4,00 & 0,60 & 11,00 & & & & 4,20 & 0,70 & 1 \\
\hline Ephén & 3,10 & 7,60 & 28,00 & 27,70 & 4,36 & 33,00 & 0,60 & 1,50 & 23,00 & 30,80 & 5,75 & 36 \\
\hline Coléoptères & 0,40 & 1,32 & 5,40 & 0,10 & - & 5,60 & 0,01 & & 6,00 & & - & \\
\hline $\begin{array}{l}\text { Hétérop. } \\
\text { Diptères }\end{array}$ & 640 & 0,53 & 46,00 & 0.20 & _ & 9,00 & 19,20 & 0,96 & 46,00 & 0,20 & - & 10 \\
\hline $\begin{array}{l}\text { Chironomes } \\
\text { autres } \\
\text { Poissons }\end{array}$ & $\begin{array}{c}19,70 \\
0,20 \\
0,54\end{array}$ & 4,35 & $\begin{array}{c}55,00 \\
6,60 \\
5,40\end{array}$ & $\begin{array}{c}52,20 \\
8,00 \\
2,00\end{array}$ & $\begin{array}{c}0,73 \\
0,11 \\
86,00\end{array}$ & $\begin{array}{l}41,00 \\
17,00 \\
20,00\end{array}$ & $\begin{array}{c}5,80 \\
31,40 \\
1,00\end{array}$ & $\begin{array}{l}0,74 \\
4,05 \\
90,40\end{array}$ & $\begin{array}{l}46,00 \\
23,50 \\
12,00\end{array}$ & $\begin{array}{c}34,40 \\
27,80 \\
2,30\end{array}$ & $\begin{array}{r}0,60 \\
0,40 \\
92,90\end{array}$ & $\begin{array}{l}80 \\
55 \\
41\end{array}$ \\
\hline
\end{tabular}

Les perches de taille inférieure oli égale à $10 \mathrm{~cm}$ capturent un très large éventail de proies, depuis les Copépodes Cyclopoldes ou les ostracodes, jusqu'aux alevins de Cyprinidés. Le rapport entre les biomasses de la plus petite et de la plus grande de ces proies est de 1 'ordre de 1 à $3.10^{4}$. La comparaison des fréquences relätives des proies, de leurs occurrences ou des biomasses relatives ne donne pas une image correcte de leur importance dans l'alimentation de la perche. si on considère seulement la fréquence ou l'occurrence des proies, le plancton et les larves de diptẻres constituent, été comme hiver, l'essentiel de $l^{\prime a l i m e n t a t i o n ~ d e s ~ j e u n e s ~ p e r c h e s, ~ e t ~ l a ~ b i o m a s s e ~ t x e ̀ s ~ i m p o r t a n t e ~ c o n s t i-~}$ tuée par les alevins de cyprinidés est négligée. Les valeurs pondérées de l'indice I.R.I. sont beaucoup plus représentatives de la situation. Elles sont en hiver, de 2546 pour le plancton, 1322 pour les Chironomes, 428 pour les poissons, 300 pour les Ephêmères et les Hétéroptères corixidae, et inférieures à 10 pour les autres proies. En été l'importance relative du plancton diminue (IRI $=1635$ ) alors que celle des poissons, 
des Diptères et des Hétéroptêres s'est accrue de façon à peu près équivalente : IRI voisins de 1000 pour ces trois groupes.

L'étude détaillée de la fraction planctonique des contenus stomacaux des sujets de 4 à $10 \mathrm{~cm}$, en hiver (tableau 2), montre que les cladocères Sida cristallina et Simocephalus vetulus, le copépode Macrcyclops albidus représentent 85,5 \& des proies de cette catégorie et 98,6 \& de la biomasse planctonique conscmmée par les jeunes perches. En raison de leur taille, les deux cladocères sont beauccup mieux représentés dans les contenus stomacaux que dans le plancton, surtout pendant la saison froide.

En hiver, les larves de Chironomes, puis les poissons et les Ephéméroptères sont les proies les mieux représentées dans les contenus stomacaux des perches de plus de $10 \mathrm{~cm}$ avec des valeurs du I.R.I. qui sont respectivement de 2 173, 1 760, 1 057. La valeur pour le plancton à cette période est de 18. Durant la saison chaude, l'ichtyophagie est encore plus marquée (IRI $=3860$ pour les alevins de Cyprinidés) mais les Chironomes et les Ephéméroptères sont encore présents dans presque tous les estomacs avec des valeurs respectives de 2800 et 1315 . En certains points particuliers de la retenue, les estomacs de perches de grande taille $(35-45 \mathrm{~cm})$ contiennent le plus souvent des Décapodes du genre Astacus.

Tableau 2.-. Composition spécifique de la fraction planctonique des contenus stomacaux des perches de petite tạile (Lf $<10 \mathrm{~cm})$, dans le lac de sainte-Croix en hiver.

Table 2.- Planctonic prey in stomach content of small perch (Lf $<10 \mathrm{~cm}$ ) in winter, in the Sainte-Croix reservoir.

\begin{tabular}{llll}
\hline & $\begin{array}{l}\text { taille fréquence } \\
\text { mm. Pi \% }\end{array}$ & $\begin{array}{l}\text { biomasse } \\
\text { Mi \% }\end{array}$ \\
\hline Cladocères & & & \\
Sida cristalina & 5 & 33,2 & 79,2 \\
Daphnia sp. & 0,5 & 0,5 & \\
Simocephalus vetulus & 2 & 10,6 & 5,4 \\
Bosmina longirostris & 0,5 & 0,5 & \\
Acroperus harpae & 0,8 & 1,1 & \\
Biapertura affinis & 1 & 0,1 & \\
Chydorus sphaericus & 0,4 & 0,1 & \\
Copepodes & & & \\
Macrocyclops albidus & 2 & 41,7 & 14 \\
Eucyclops serrulatus & 1,1 & 0,8 & \\
Eucyclops macrurus & 1,1 & 2,7 & 0,2 \\
Acanthocyclops robustus & 1,3 & 5,6 & 0,4 \\
Cyclops vicinus vicinus & 1,9 & 3 & 0,6 \\
\hline
\end{tabular}

\section{2 - Croissance linēaire et pondérale}

Les tailles moyennes rétrocalculées pour chaque classe d'âge suivant le modèle logarithmique (tableau 3), sont en concordance, à un cm près, avec les tailles observées pour les quatre premières années de vie. Bien que l'effectif soit peu élevé, on ne note pas de classes d'age vides, corsécutives à l'échec d'une année de reproduction par exemple. 
Tableau 3.- croissance linéaire rétrocalculée dans un échantillon de 126 perches capturées dans le lac de Sainte-croix.

Table 3.- Back calculated length for 126 perch fished in the Sainte-Croix reservoir.

\begin{tabular}{|c|c|c|c|c|c|c|c|c|c|c|}
\hline \multirow[t]{2}{*}{ Cohortes } & \multirow[t]{2}{*}{ Age } & \multicolumn{2}{|c|}{ observés } & \multirow[t]{2}{*}{ Nombre } & \multirow{2}{*}{$\begin{array}{c}\text { Lf. } \\
\text { I }\end{array}$} & \multirow{2}{*}{$\begin{array}{c}\text { moy. } \\
\text { II }\end{array}$} & \multicolumn{3}{|c|}{ retrocalculées } & \multirow[b]{2}{*}{ VI } \\
\hline & & Lf $\mathrm{cm}$ & $\mathbf{P g}$ & & & & III & IV & V & \\
\hline 1984 & 1 & 7,9 & 6,5 & 30 & 8,8 & & & & & \\
\hline 1983 & 2 & 10,0 & 14,0 & 36 & 8,3 & 11,6 & & & & \\
\hline 1982 & 3 & 14,0 & 39,0 & 20 & 8,3 & 11,6 & 14,1 & & & \\
\hline 1981 & 4 & 16,3 & 61,0 & 17 & 8,3 & 11,1 & 13,8 & 15,7 & & \\
\hline 1980 & 5 & 18,5 & 87,0 & 13 & 8,1 & 11,0 & 13,3 & 15,6 & 17,1 & \\
\hline 1979 & 6 & 20,7 & 127,0 & 10 & 8,1 & 10,5 & 12,9 & 15,0 & 16,8 & 18,7 \\
\hline Lf. & & & & & 8,4 & 11,4 & 13,8 & 15,8 & 17,0 & 18,7 \\
\hline
\end{tabular}

Les 2 plus grands sujets capturés ont été des femelles âgées de 9 à 10 ans qui mesuraient 40 et $44 \mathrm{~cm}$ et pesaient respectivement $1400 \mathrm{~g}$ et $1710 \mathrm{~g}$. De tels individus sont rares et ils n'ont pas été pris en compte dans le rétrocalcul.

La relation entre la taille à-la fourche exprimée en cm et le poids en $g s^{\prime}$ écrit :

$$
P=108.10^{-4} \text { Lf }^{3.102} \quad r=0.99 \quad n=180
$$

Comparée à quelques donnêes de la bibliographie (figure 2), la croissance linéaire observée a sainte-croj.x est assez faible, inférieures à celles qu'observent JELLYMAN (1980) sux le lac Pounul, CRAIG (1974) sur le slapton Ley et DAUBA (1981) sur la retenue de Chastang. Elle est par contre plus rapide que sous les hautes latitudes (SUMARI, 1971).

\section{3 - Fécond itẽ}

Au terme de la première année, 75 qes mâles sont à maturité sexuelle, alors que les femelles les plus précoces, 60 \% environ, ne pondent qu'à l'âge de deux ans. Tous les individus mâles et femelles de trois ans sont sexuellement murs.

La relation liant la fécondité absolue à la longueur dans un lot de 15 femelles de 14 à $44 \mathrm{~cm}$ dont les ovaires contenaient 2500 à 50 ooo ovules a été calculée :

$$
\text { Fec }=0,6857 \mathrm{~L}^{2,510} \quad r=0,94 ;
$$

La relation poids-fécondité s'écrit $\mathrm{Fec}=2,2770 \mathrm{p}^{0,0092}$. La fécondité de la perche dans le lac de ste-croix est réduite. Pour les sujets de petite taille, elle est du meme orare que celle decrite par JELLYMAN (1980), mais encore plus basse pour les femelles de grande taille (tableau 4). La fécondité relative, exprimée en nombre d'oeufs par gramme de poids somatique, pour les femelles pesées après ablation des ovaires, est une fonction décroissante du poids. Dans l'échantillon que noụs avons analysé elle présente une grande variabilité (tableau 3), mais les $2 / 3$ des valeurs sont comprises entre 32 et 95 , ce qui les range parmi les plus faibles de la littêrature. 
$\mathbf{L}_{\mathrm{t}} \mathrm{mm}$.

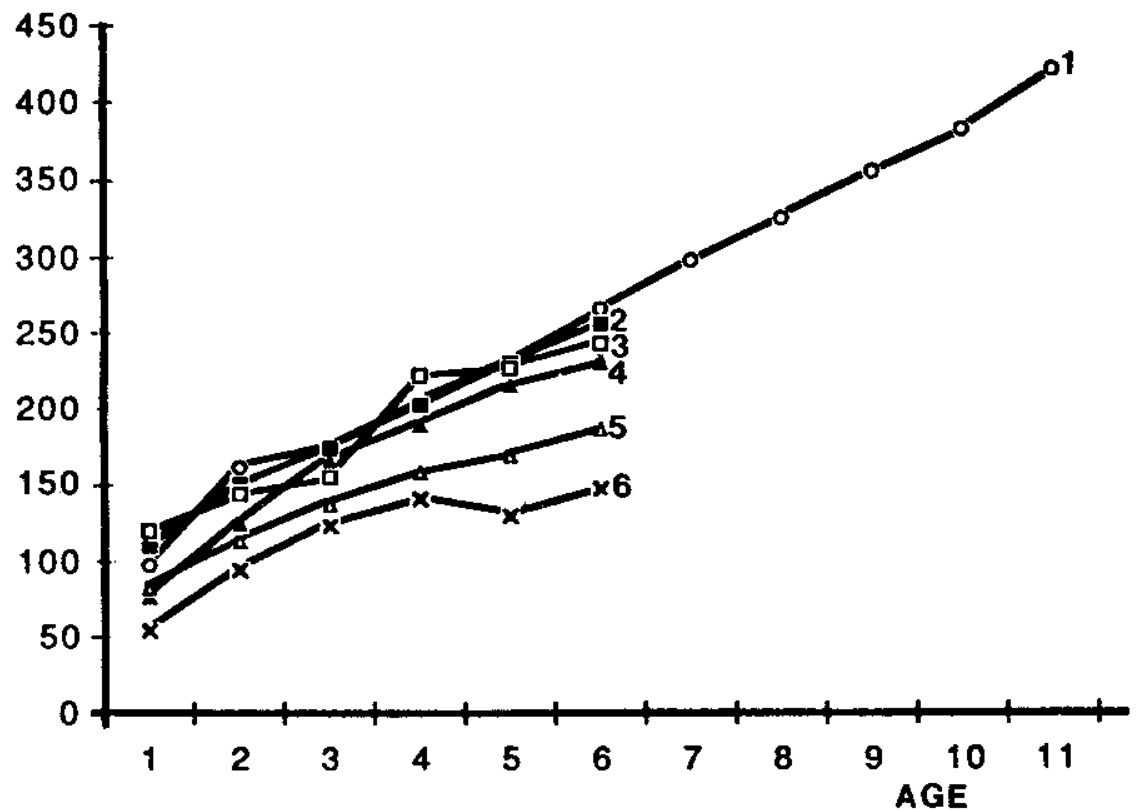

Figure 2.- Croissance linéaire de la perche à sainte-Croix (5). Comparaison avec quelques données de la littếrature.

Figure 2.- Growth of perch in the Sainte-Crcix reservoir (5), and comparison with growth in some other localities :

(1) Lake of the Woods, JOBES, 1952 in THORPE, 1977 ;

(2) Lake Pounui, JELLYMAN, 1980 ; (3) Chastang reservoir, DAUBA, 1981 ; (4) Slapton lay, CRATG, 1974 ; (6) Pond in Fintand, SUMARI, 1971 in THORPE, 1977.

Tableau 4.- Fécondité de la perche dars le lac de Sainte-Crcix ; comparaison avec quelques données de la littérature.

rable 4.- Perch fecondity in the Sainte-Croix reservoir; comparison with literature data.

\begin{tabular}{llcrr}
\hline Localités & \multicolumn{1}{c}{ Auteurs } & L cm & Fec. absolue & Fec.relative \\
\hline Baltic sea & ANEER, GRAHN & $19-36$ & $6500-85000$ & $50-156$ \\
Loch Leven & THORPE 1977 & $23-31$ & $26200-61300$ & $80-156$ \\
Klicava reservoir & STEHLIK 1968 & $12-23$ & $6710-14400$ & $91-317$ \\
Lac Vortsjärv & PIHU 1964 & $12-34$ & $6380-93500$ & $118-397$ \\
Loch Davan & TREASURER 1981 & $14-34,5$ & $6711-77978$ & \\
Lac Pounui & JELLYMAN 1980 & $14-42$ & $2657-63858$ & $33-141$ \\
Slapton Ley & CRAIG 1974 a & $10-28$ & $1000-30500$ & $52-188$ \\
Lac Dojran & PETROVSKI 1960. & $15-33$ & $4370-81801$ & $70-202$ \\
Lac Sainte Croix & étude présente & $15-44$ & $2500-50000$ & $32-334$ \\
\hline
\end{tabular}




\section{4 - Discussion}

LE CREN (1958), GOLDSPINK et GOLDWIN (1979), ont montré l'importance des facteurs climatiques dans la réussite et le développement des alevins de perches. Il existe, dans le lac windermere par exemple, une corrélation positive entre le nombre de degrés-jours au dessus de $14{ }^{\circ} \mathrm{C}$ pendant l'année et l'accroissement en taille durant la même période. ERIKSSON (1978), montre que l'activité de la perche est en relation positive avec la durée du jour et la température.

Au point. de vue thermique, le lac de Ste-croix offre des conditions favorables avec plus de 900 degrés-jours au dussus de $14{ }^{\circ} \mathrm{C}$, soit le double du lac de windermere.

En Europe du nord, la perche semble caractéristique des lacs oligotrophes, tandis que le gardon est dominant daris les eaux eutrophes et mésotrophes (HARTMAN, 1977 ; LESSMARK, 1983). Il s'agtt de lacs oũ la température estivale est plus faible que celle enregistrée à ste-Croix, ce qui peut expliquer la dominance du gardon sur la perche dans la retenue oligotrophe de ste-Croix.

En effet, le taux de croissance maximum est plus élevé pour la perche que pour le gardon lorsque les températures sont basses. Pour un poisson de $10 \mathrm{~g}$, la température optimale est de $18^{\circ} \mathrm{C}$ chez la perche et de $20^{\circ} \mathrm{C}$ chez le gardon (LESSMARK, 1983). Ainsi, la température qui favorise le đéveloppement des alevins et des adultes de pexches, favorise encore davantage le développement des populations de gardons. Depuis une dizaine d'années, nous avons assisté à l'explosion des populations de gardons et d'ablettes qui colonisent toute la retenue de ste-Croix.

L'étude des contenus stomacaux mortre que les jeunes perches se nourrissent à ste-Croix, aux dépens d'un zooplancton de grande taille. BOULET (1958), dans une étude expérimentale, indique que la perche jouit d'une bonne perception visuelle diurne du mouvement, pourvu que les objets soient assez volumineux ; la plupart des sujets testés, sont indifférents au passage d'une sphère de $2 \mathrm{~mm}$, sauf si elle est animée d'une trajectoire complexe en "nage de daphnies", fortement stimulante, malgré l'absence de facteurs chimiques et vibratoires. LESSMARK (1983), incique que les proies capturées par la perche ne sont jamais inférieures a $0.4 \mathrm{~mm}$, KLEMETSEN (1973) donne une taille minimum de 1,2 m pour les proies. Cependant, comme le souligne CRAIG (1974), la disponibilité d'une proi.e est très importante dans le déterminisme de sa capture.

La perche est un prédateur diurne (GUMA'A, 1982 ; DABROWSKY, 1982), parfois crépusculaire (KOGAN et al., 1981). Chasseur visuel, qui consomme de grandes quantités d'énergie dans la recherche de sa nourriture, elle apparalt moins bien adaptée au régime planctonophage que le gardon et 1'ablette, chasseurs peu actifs dans la recinerche de leur nourriture et capables de se nourrir dans l'obscurité grăce aux perceptions de leur ligne latérale (DABRONSKT, 1982).

HOLCIK (1966, 1970), IVANOVA (1953), observent un développement rapide des populations de perches immédiaterent après la mise en eau d'une retenue, suivi d'une reduction d'effectifs en relation avec le développement du gardon et de 1'ablette. De même, un développement de la population. de perches est observée après réduction d'effectifs chez le gardon (PERSSON, 1986). 
PERSSON (1983, 1987), STENSON (1979), EIE et BORGSTROM (1981), montrent que l'alevin de perche se nourrit du méme zooplancton que I'aievin de gardon ; l'activité prêdatrice de ces deux espèces s'exerce d'abord au détriment des zooplanctontes de grande taille, favorisant ainsi les formes de petite taille que le gardon est plus apte à capturer que la perche. L'ablette, seul planctonophage adulte, dans le lac de Sainte-Croix, consomme égalemert les plus grosses daphnies (CHAPPAZ et al., 1987). PONT et $a$., (1988) observent depuis quelques années une diminution de la taille moyenne des daphnies, dominantes dans la retenue de Sainte-Croix, en relation avec le développement de la population d'ablettes.

Dans le lac Sovdeborgssjon, Daphnia sp. est. peu reprësentée sous l'effet de la prédation par le gardon et la pénurie de gros zooplanctontes contraint les juvéniles de perches à s'alimenter à partir des macroinvertébrés (PERSSON, 1983).

Il en est de même dans le lac de ste-Croix oũ les Ephémères et les chironomes participent à l'alimentaticn des perches de toutes tailles. Dans ces conditions le chevauchement. des niches alimentaires des juvëniles et les adultes favorise la compétition intraspécifique.

La disponibilité des proies benthiques est largement conditionnée par la gestion hydraulique de la retenue. Le marnage hivernal met à sec 23 de la surface totale du lac qui constituent, en fait, l'essentiel de la zone exploitée par les poissons. Une campagne d'ếchosondage-échointégratior a en effet montré qu'en situation non marnée la densité des poissons chute trẽs rapidement lorsqu'on s'élolgne des berges. Les Chironomes comme les olirgochètes présentent des formes de résistance à I'assec, dernier stade larvaire chez les Chironomini ou adultes dans un kyste muqueux chez Lumbricullus variegatus (CHAMPEAu et al., 1980), qui leur permettent de se maintenir dans le milieu. Le marnage en diminue cependant la quantité disponible pour les poissons, interdit le développement. d'espèces dépourvues de stade de résistance, comme il limite I'abondance des larves d'insectes et du plancton liés à l'herbier. Myriophylles et potamots ne présentent de tiges feuillées que durant quelques mois seulemert, même s'ils supportent l'assec gráce à leurs rhizomes. Le benthos exploitable par les perches est donc en situatior. de survie pendant une grande partie de l'année ce qui en interdit une densité plus élevée.

Les alevins de Cyprinidés sont présents en toutes saisons dans les estomacs des perches capturées même chez les sujets de taille infêrieure à $10 \mathrm{~cm}$. Leur importance dans le régime alimentaire de cette espèce augmente fortement en été avec l'apparition des nouvelles cohortes d'ablettes et de gardons qui accroissent la disponibilité en proie de petites tailles accessibles aux juvéniles.

Des cas de croissance ralentie sont observés dans des milieux de petites dimensions où la compétition intraspécifiçue est intense (HOESTLAND, 1979 ; BOUJARD, 1987). La croissance linéaire lente mesurée dans le lac de Ste-Croix, trouve son origine à la fois dans la compétition intraspécifique provoquée par le chevauchement des niches alimentaires des perches de tailles différentes, et par la compétition interspécifique avec le gardon et 1 'ablette. Ces conditions trophiciues difficiles retentissent aussi sur la fécondité qui prenđ des valeurs parmi les plus basses de la litterature. En termes de gestions piscicoles il apparait difficile de développer cette espèce dans le lac de Ste-Croix dans les conditions actuelles. 


\section{REMERCIEMENTS}

Les auteuxs remercient $D$. PONT pour la détermination de la fraction planctonique des contenus stomacaux, Ph. BOET pour ses critiques et suggestions, ainsi que Messieurs les présidents des Fédérations AAPP et les Membres de la garderie des Alpes de Haute provence et du Var.

Ce travail a bénéficié de l'aide financière du C.N.R.S. (GRECO 81, Fonctionnement des Ecosystèmes lacustres) et d'Electxicité de France, R.E.A.M.

\section{RÉFÉRENCES BIBLIOGRAPHIQUES}

BOUJARD T. (1987). Mise en Évidence de deux groupes d'individus aux caractéristiques de croissance et de comportement distinctes au sein d'une population de perches (Perca fluviatilis L.) dans un étang de Bretagne. Acta Oecologica, Oecol. Applic., 8(3): 179-189.

BOULET P.C. (1958). Contribution a l'étude experimentale de la perception visuelle du mouvement chez la perche et la selche. Mém. Mus. Natl. Hist. Nat. Paris, Ser. A. 2001., 17, 131 p..

BRESSAC Y, (1985). Le zooplancton des lacs de barrage. Les retenues du sudest de la France. Influence des arrivées d'eau et de la qualité du seston sur la structure spaciale du peuplement. These doct. Universite de Provence, 144 p..

CHAPPAZ R., BRUN G., OLIVARI G. (1987). Mise en évidence de differrences de régine alimentaire dans une population d'ablettes. Alburnus alburnus (L.) dans le lac de ste-crolx. Conséquences sur la fécondité. Annle timnol., $23(3)$ : 245-252.

CHAMPEAU A. et al., (1982). Les retenues hydroelectriques du verdon : impact sur la rivière, consequences du marnage. Bulz. Ecol., 13(2): 203-239.

CRAIG J.F. (1974). Population dynamics of perch, Perca fluviatilis in slapton hey Devon.

a) I. Trapping behaviour, reproduction, migration, population estimates, mortality and food. Freshwater Biol., 4 ; 417-431. b) II. Age, growth, lenght, weight relationships and conditions. Freshwater Biol., 4: 433-444.

DABROWSKY K.R. (1982). The influence of light intensity on feeding of fishes larvae and fry. Zool. Jahrb. Abt. Allg. Zool. Fhysiol. Tiere, 86(3): 353-360.
DAUBA F. (1981). Etude comparative de la faune des poissons dans les écosystèmes de deux réservoirs Luzech (Lot) et

Chastang (Dordogne). Thèse de 3ème cycle, I.N.P. Toulouse, 179 p..

EIE J.A., BORGSTROM R. (1981). Distribution and food of roach Rutilus rutilus $L$. and perch Perca fluviatilis $L$. in the eutrophic lake Arungen, Norway. Verh. Int, Ver. Theor. Angew. Limmol., 21: $1257-1263$.

EL HAFA M. -(1987). Bilan des apports nutritifs au lac de ste-croix et leur utilisation par le phytoplancton de la retenue. Thèse jème cycle. Université de Provence, 131 p.

ERIKSSON (1978) in NEUMAN (1979). Activity of perch Perca fluviatilis $L$. and roach Rutilus rutilus $\mathrm{s}$. In a Baltic Bay with special reference to temperature. Rep. Inst. Freshw. Res. Drottningholn, 58: $107-125$.

GOLDSPINK C.R. (1979). The population density growth rate and production of roach Rutilus rutilus $L$. in $T$ jeukemer. The Netherlands. J. Fish. Biol., 15(4): 473-49日.

GOLDSPINK C.R. et GOLDWIN D. (1979). A note on the age composition growth rate and food of perch Perca fluviatilis $L$. in four eutrophs c lakes, England. $J$. Fish. Biol., 14: 489-505.

GUMA'A S.A. (1982). Retinal development and retinimotor responses in perch Perca fluviatilis L., J. Fish. Biol., $20(5)$ : $611-618$.

HARTMANN J. (1977). Fische reiliche Veranderungen in kultubedingt eutrophierenden Seen. Schweiz. 2. Hydrol, 39 : 243-254. 
HOESTIANDT H. (1979). Recherches biologiques sur la perche en Frar:ce. Rapport du Conseil superieur de la peche, 105 p..

HoIfIK J. (1966). Vyraj a formovanie ichtyofauni v Oravskej priehrade, Biol. Pr. Brats $2,12(1): 5-75$

(1970), Klicava reservoi.r an ichtyologj.cal study, Biol. Pr. Bratst, 15(3): 5-94.

HYSIOP E.J. (1980) . Stomach content analysis - a review of methods and the1r appl1cations. J. Fish Biol., 17: 411-429.

IVANOVA M.N. (1953). Blologicheskie osobennosti plotvy kak komponenta ozernoi ikhtiofauny. Vopr. Ikhtiol., 1: 82-93.

JELLYNAN D.J. (1980). Age, growth and reproduction of perch Perca fluviatilis $\mathrm{L}$. in lake Pounui. N.Z. Jowmal of mamine and frestuxater research, 14(4): 391-400.

KLEMETSEN A. (1973). Pelagic planctor. eating Perch. Astarte, 6, 27-33.

KOGAN A.V., NEBRASOVA N.P., POPOVA I.K. (1981). Feeding peculiarities of the young of the roach Rutilus rutilus L. and perch, Perca fluviatilis during downstream migration. USSR Academy of sciences, Moscou: 106-113.

IE CREN E.D. (1947). The determination of the age and growth of the perch Perca fluviatilis from the opercular bones. $J$. Anim. Ecol., 16: 188-204.

LE CREN E.D. (1958). Observation of the growth of the perch over twenty two years with special reference to the effects of temperature and changes in population density. J. Anim. Ecol. 27.

IEssmark O. (1983). Competition between perch Perca fluviatilis and roach Rutilus rutilus in south Swedish lakes. Doctoral dissertation 172 p., Universite LUND.
PONT D. CHAPPAZ R, CHAMPEAU A, BRUN G. (1988). Dynamique d'un système proies-prédateurs (zooplancton Poisson) dans une retenue artificielle de mise en eau récente (Sainte-croix PROVENCE, FRANCE). II. Conf. Int. Limnologues Expression Française.

PERSSON L. (1983). Food consumption and competition between age classes in a perch Perca fluviatilis population in a shallow eutrophic lake. Oikos DNK, $40(2): 197-207$.

PERSSON L. (1986), Effects of reduced interspecific competition on ressource utilization in perch Perca fluviatilis. Ecology, $67(2)$ : 355-364.

PERSSON I. (1987). Competition induced switch in young of the year perch Perca fluviatilis : an experimental test of ressource limitation. Envir. Biol. of Fishes, 19(3): 235-239.

SIMPSON (1951). The fecundity of the plaice. Fishes Invest. Londun, $17(5)$ : 133-146.

STEHLIKJ (1968). The fecundity of pexch Perca fluviatilis in the klicava water reservoir. Vestn. Cesk. Spol. Zool., $33(1)$ : $88-95$

STENSON J.A. (1979). Perdator-prey relation between fish and invertebrate pxey in some forest lakes. Report from the Swedish State Institute of freshwatex Research, Drottningholm, 58: 166-183.

SUMART O. (1971). Structure of the perch populations of some ponds in Finland. Ann. Zool. Fenn., $8(3): 406-421$.

THORPE J. (1971). Synopsis of blological data on the perch Perca fluviatilis and Perca flavescens (H1tchill, 1814). F.A.O. Fisheries Symopsis, $n^{\circ} 113$.

TREASURER J.W. (1981) . Some aspects of the reproductive biology of perch Perca fluviatilis $L$. Fecundity, maturation and spawning behaviour. J. Fish. Biol., 18: 729-740. 